\title{
Cobertura vacinal contra a Hepatite B dos profissionais de saúde do Centro de Saúde de Queluz
}

Joana Severo de Almeida,* Susana Gomes*

\section{RESUMO}

Introdução: A Hepatite B é uma importante causa de morbilidade e mortalidade a nível mundial, existindo transmissão relacionada com exposição ocupacional. A vacinação contra a hepatite B induz protecção elevada, encontrando-se incluída no Plano Nacional de Vacinação e prevista para grupos de risco, nomeadamente para os profissionais de saúde.

Objectivo: Determinar a prevalência da vacinação contra a Hepatite B nos profissionais de saúde do Centro de Saúde de Queluz. Tipo de estudo: Descritivo, transversal, observacional.

Local: Centro de Saúde de Queluz

População: Profissionais de saúde pertencentes a grupos de risco

Métodos: Os dados foram obtidos através do Sistema de Informação para as Unidades de Saúde (SINUS) ou da consulta directa do Boletim Individual de Saúde e na ausência destes por inquérito aos profissionais. Foram consideradas as variáveis sexo, idade, profissão, estado vacinal relativamente à hepatite $B$.

Resultados: A população foi constituída por 132 indivíduos, obtendo-se resultados respeitantes a 104 indivíduos. Entre estes, $81,7 \%$ apresentaram vacinação completa, 5,8\% incompleta e 12,5\% não apresentaram qualquer vacinação.

Apresentaram uma vacinação completa $92,1 \%$ dos enfermeiros, $79,7 \%$ dos médicos e $42,9 \%$ dos auxiliares. Os auxiliares de acção médica foram o grupo com maior percentagem de ausência de vacinação - 28,6\%, seguidos dos médicos - 16,9\% e dos enfermeiros $-2,6 \%$.

Conclusões: Será importante avaliar se estes dados revelam, efectivamente, baixas taxas de cobertura vacinal contra a hepatite B nos profissionais de saúde, alargando este estudo a outros profissionais de outros centros de saúde. Dada a indicação clara no sentido da vacinação destes profissionais, poder-se-á actuar junto dos indivíduos não vacinados de modo a veicular informação de forma perceptível, enfatizando os benefícios obtidos com a prevenção primária. Contudo, deverá ser ponderada a possibilidade de realização de futuros estudos, no sentido de avaliar os motivos que levam a esta não vacinação.

Palavras-Chave: Vacinação; Hepatite B; Profissionais de Saúde.

\section{INTRODUÇÃO}

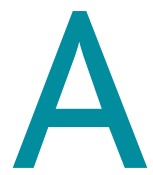

hepatite B é uma importante causa de morbilidade e mortalidade a nível mundial, dada a sua tendência para causar infecção persistente, hepatite crónica e a possibilidade de evolução para carcinoma hepatocelular. ${ }^{1,2} \mathrm{~A}$ incidência desta neoplasia, na Europa, em indivíduos com cirrose, é de 2 a 6\%/ano ao fim de cinco anos de doença. ${ }^{3}$

*Assistente Eventual de Medicina Geral e Familiar no Centro de Saúde de Queluz - Unidade de Saúde Familiar de Mactamã
Estima-se que exista seropositividade para a hepatite B, fora de um contexto de vacinação, em cerca de um terço da população mundial. ${ }^{2,3} \mathrm{Na}$ Europa Ocidental observa-se uma prevalência de Hepatite B de $2 \%$ a $7 \%$ existindo, na região sul da Europa, uma incidência anual aproximada de 6\%., Portugal é um país de endemicidade baixa a intermédia, com cerca de 1,4\% de portadores crónicos. ${ }^{3}$ Nos Estados Unidos da América (EUA) foram identificados, entre 1984 e 1993, 53.000 novos casos, entre os quais 8.000 eram relacionados 
com exposição ocupacional. ${ }^{5}$

A infecção pelo Vírus da Hepatite B (VHB) resulta da exposição percutânea e das mucosas a sangue e outros fluidos orgânicos nomeadamente esperma, saliva, suor ou leite de indivíduos infectados. Apresenta diversas vias de transmissão, sendo as mais frequentes a parentérica, a sexual e a vertical. ${ }^{2,3,6,7}$ Existe ainda a possibilidade de transmissão através de fluidos orgânicos como por exemplo a saliva, suor, leite, que contêm grandes quantidades de partículas de AgHbs, embora poucas partículas víricas infecciosas, reduzindo assim o risco de transmissão.,

A hepatite $B$ pode ser prevenida através de medidas específicas como a imunização. ${ }^{8} \mathrm{~A}$ vacina contra a hepatite $B$ tem eficácia e segurança elevadas, apresenta um elevado poder imunogénico e induz protecção em cerca de $90 \%$ a $97 \%$ dos indivíduos vacinados. ${ }^{1,2}$

Em Janeiro de 2000, a vacinação contra a hepatite B foi incluída no Programa Nacional de Vacinação (PNV) sendo, desde então, administrada a todos os recém-nascidos e a todos os jovens dos 10 aos 13 anos. Além da vacinação universal está ainda indicada a vacinação de grupos de risco, entre os quais destacamos o pessoal dos serviços de saúde, excluindo os que têm tarefas exclusivamente administrativas. ${ }^{9}$

Em estudos efectuados, observou-se uma prevalência de vacinação entre profissionais de saúde variável, existindo em Portugal um estudo que refere haver, entre médicos de uma instituição - Centro de Saúde de Sete Rios -, uma prevalência de vacinação de $53 \%$, embora esta fosse superior noutros grupos profissionais, ${ }^{10}$ situação também evidenciada noutros estudos fora da realidade portuguesa. ${ }^{11}$

Estes dados chamam a atenção para a eventual necessidade de acções de sensibilização junto dos grupos profissionais referidos.

Este estudo tem como objectivo determinar a prevalência da vacinação contra a hepatite B nos profissionais de saúde do Centro de Saúde de Queluz.

\section{MÉTODOS}

Foi realizado um estudo descritivo, transversal e observacional, em todos os profissionais de saúde a exercer funções no Centro de Saúde de Queluz - médicos, enfermeiros e auxiliares de acção médica, pelo que não foi criada amostra.
As variáveis estudadas foram o sexo, a idade, a profissão, o estado vacinal relativamente à hepatite $\mathrm{B}$.

O estado vacinal foi operacionalizado da seguinte forma:

- Vacinação completa - Três ou mais doses, de acordo com o esquema vacinal previsto no PNV.

- Não vacinado - Nunca efectuou nenhuma dose.

- Vacinação incompleta-Situações não incluídas nas outras categorias.

A lista de profissionais envolvidos foi fornecida pela Direcção do Centro de Saúde após pedido de consentimento para realização do estudo, tendo sido divulgada aos profissionais informação acerca da realização do mesmo.

Os dados foram obtidos através do registo informatizado do SINUS - Sistema de Informação para as Unidades de Saúde ou, na falta de dados informatizados, através da consulta directa do Boletim Individual de Saúde solicitado ao profissional. Quando não foi possível obter os dados pelo SINUS ou pelo Boletim Individual de Saúde, foi efectuado inquérito através de entrevista aos profissionais, tendo-se pedido previamente o consentimento informado destes. Foi utilizada uma folha de registo de dados (Anexo 1), preenchida para cada profissional da lista fornecida pelo Centro de Saúde. A recolha de dados decorreu durante o mês de Julho de 2007, tendo o tratamento dos dados sido feito através de uma matriz do Microsoft Office Excel.

\section{RESULTADOS}

A população foi constituída por 132 indivíduos, tendo-se obtido os resultados respeitantes a 104 indivíduos (Quadros I e II). Os dados foram obtidos por consulta do SINUS/Boletim Individual de Saúde em 57,7\% e por questionário directo em $42,3 \%$ dos indivíduos.

Entre os indivíduos estudados, $73,5 \%$ eram do sexo feminino e $26,5 \%$ do sexo masculino, com uma média de idades de 44,2 anos e mediana de 45 anos. Entre os indivíduos estudados, $81,7 \%$ apresentaram vacinação completa, 5,8\% incompleta e 12,5\% não apresentaram qualquer vacinação (Quadro III).

No que se refere aos grupos profissionais, os dados sugerem que os enfermeiros apresentaram uma vacinação completa em $92,1 \%$, os médicos em $79,7 \%$ dos indivíduos e os auxiliares em 42,9\% dos indivíduos. Os auxiliares de acção médica foram o grupo com maior percentagem de ausência de vacinação - 28,6\%, seguidos dos médicos - 


\begin{tabular}{|c|c|c|c|c|c|}
\hline & Médicos & Enfermeiros & Auxiliares & Total & $\begin{array}{c}\text { Frequência } \\
\text { relativa (\%) }\end{array}$ \\
\hline Respondeu & 59 & 38 & 7 & 104 & 78,79 \\
\hline Não respondeu & 16 & 11 & 1 & 28 & 21,21 \\
\hline Total & 75 & 49 & 8 & 132 & 100,00 \\
\hline
\end{tabular}

$\begin{aligned} & \text { QUADRO II. Distribuição da população de acordo com o grupo profissional, } \\
& \text { sexo e idade }\end{aligned}$
\begin{tabular}{l|c|c|c|c|c|c|}
\hline $\begin{array}{l}\text { Mrupos etários/ } \\
\text { ISexo }\end{array}$ & Feminino & Masculino & Feminino & Masculino & Feminino & Masculino \\
\hline $20-29$ anos & 6 & 2 & 3 & 0 & 0 & 0 \\
\hline $30-39$ anos & 7 & 5 & 11 & 3 & 0 & 1 \\
\hline $40-49$ anos & 10 & 8 & 26 & 2 & 3 & 0 \\
\hline $50-59$ anos & 21 & 12 & 4 & 0 & 3 & 0 \\
\hline$\geq 60$ anos & 2 & 2 & 0 & 0 & 1 & 0 \\
\hline
\end{tabular}

16,9\% e dos enfermeiros - 2,6\%. (Quadro IV).

Em termos de distribuição por idade, entre os dados obtidos sobre a vacinação completa, a percentagem foi semelhante dos 20 aos 49 anos ( $87,5 \%$ a $89,2 \%)$, mas inferior no grupo etário dos 50-59 anos (65,5\%) (Quadro V).

\section{DISCUSSÃO}

Os resultados deste estudo correspondem a 78,8\% dos profissionais do Centro de Saúde de Queluz. Os restantes não foram incluídos por se encontrarem ausentes do Serviço-7 indivíduos -, ou por desconhecerem o seu estado vacinal - 21 indivíduos. De referir que nenhum indivíduo recusou participar no estudo.

Durante a realização deste trabalho foi notória a dificuldade na obtenção dos Boletins Individuais de Saúde, pelo que houve necessidade de recorrer, por diversas ocasiões, ao questionário directo. Este facto poderá ter condicionado um viés de informação pela possibilidade de indução de resposta, dado não se tratar de um questionário anónimo. Por outro lado, a fiabilidade da informação recolhida, por terem sido utilizados métodos diferentes de recolha de dados, está dependente, no questionário directo, da memória dos intervenientes relativamente à vacinação. No entanto, esta forma de obtenção de dados não ocorreu na maior parte dos casos.

A percentagem de respostas ao estudo foi elevada. Este facto poder-se-á dever à realização de questionários directos em $42,3 \%$ dos indivíduos, o que poderá ter motivado a sua maior adesão.

Os enfermeiros constituem o grupo profissional que apresentou maior percentagem de vacinação completa $(92,1 \%)$. No que se refere ao grupo dos médicos, $79,7 \%$ apresentaram vacinação completa. Estes valores são superiores aos apresentados noutros trabalhos, entre os quais um realizado no Centro de Saúde de Sete Rios $^{10} \mathrm{em} 1996$, em que estes resultados foram de $74,1 \%$ e $47,0 \%$, respectivamente. A variação encontrada entre estes dois estudos, poderá sugerir que nos 11 anos que decorreram entre ambos, poderá ter havido não só uma maior divulgação da importância da vacinação, como também uma maior consciencialização da mesma, junto destes grupos profissionais. Para esclarecer as causas que motivaram esta diferença deverão ser realizados estudos posteriores.

O grupo profissional que parece apresentar a maior percentagem de não-vacinação é o dos auxiliares de acção médica $(28,6 \%)$ - dois indivíduos entre um total 


\section{QUADRO III. Estado vacinal dos profissionais de saúde} do Centro de Saúde de Queluz

\begin{tabular}{l|c|c} 
& $\begin{array}{c}\text { Número de } \\
\text { indivíduos }\end{array}$ & $\begin{array}{c}\text { Frequência } \\
\text { relativa (\%) }\end{array}$ \\
\hline Vacinação completa & 85 & 81,73 \\
\hline Vacinação incompleta & 6 & 5,77 \\
\hline Sem vacinação & 13 & 12,50 \\
\hline Total & 104 & 100,00
\end{tabular}

QUADRO IV. Estado vacinal distribuído por grupo profissional

Médicos Enfermeiros Auxiliares

\begin{tabular}{l|r|r|r}
\hline Vacinação completa & 47 & 35 & 3 \\
\hline Vacinação incompleta & 2 & 2 & 2 \\
\hline Sem vacinação & 10 & 1 & 2 \\
\hline Total & 59 & 38 & 7
\end{tabular}

\section{QUADRO V. Percentagem da população com vacinação completa de acordo com o grupo etário}

\begin{tabular}{l|c|c|c} 
& $\begin{array}{c}\text { Percentagem de indivíduos } \\
\text { com vacinação completa }\end{array}$ & $\begin{array}{c}\text { Percentagem de indivíduos } \\
\text { com vacinação incompleta }\end{array}$ & $\begin{array}{c}\text { Percentagem de indivíduos } \\
\text { não vacinados }\end{array}$ \\
\hline $20-29$ anos & $87,50 \%$ & $0,00 \%$ & $12,50 \%$ \\
\hline $30-39$ anos & $88,46 \%$ & $3,85 \%$ & $7,69 \%$ \\
\hline $40-49$ anos & $89,19 \%$ & $8,11 \%$ & $2,70 \%$ \\
\hline $50-59$ anos & $65,52 \%$ & $6,90 \%$ & $27,59 \%$ \\
\hline $\mathbf{2 0}$ anos & $75,00 \%$ & $0,00 \%$ & $25,00 \%$ \\
\hline
\end{tabular}

de sete. Este valor é semelhante ao encontrado no estudo já referido $(20 \%) .^{10}$

Dada a indicação clara no sentido da vacinação nestes profissionais poder-se-á actuar juntos dos grupos profissionais que apresentaram maior percentagem de não vacinação, tendo em conta as suas características específicas, de modo a veicular informação de forma perceptível, em cada grupo envolvido, enfatizando de forma objectiva e clara os benefícios obtidos com a prevenção primária.

Será importante avaliar se estes dados revelam, efectivamente, baixas taxas de cobertura vacinal contra a hepatite B nos profissionais de saúde, alargando este estudo a outros profissionais de outros centros de saúde e melhorando o método de recolha desses dados. Poder-se-á ponderar a realização de futuros estudos no sentido de avaliar os motivos que levam a esta não vacinação.

\section{REFERÊNCIAS BIBLIOGRÁFICAS}

1. Lemon SM, Thomas DL. Vaccines to prevent viral hepatitis. N Engl J Med 1997 Jan 16; 336 (3): 196-204.

2. Ministério da Saúde; Direcção Geral de Saúde. Avaliação do Programa Nacional de Vacinação e melhoria do seu custo-efectividade: $2^{\circ}$ inquérito serológico nacional: Portugal Continental 2001-2002, principais resultados. Lisboa: DGS; 2004.

3. Marinho C, Agostinho C. Hepatite B. In: Cotter J, editor. Hepatites Víricas (monografia na Internet). Núcleo de Gastroenterologia dos Hospitais Dis- tritais. p. 53-98. Disponível em: http://ww.aidsportugal. com/hepatites/3_8.pdf [acedido em 28/11/2009].

4. Roure C. Overview of epidemiology and disease burden of hepatitis B in the European region. Vaccine. 1995; 13 Suppl 1: S18-21.

5. Barash C, Conn M, DiMarino AJ Jr, Marzano J, Allen ML. Serologic hepatitis B immunity in vaccinated health care workers. Arch Intern Med 1999 Jul 12; 159 (13): 1481-3.

6. Poland CA, Jacobson RM. Clinical practice: prevention of hepatitis B with the hepatitis B vaccine. N Engl J Med 2004 Dec 30 ;351 (27): 2832-8.

7. Murray PR, Rosenthal KS, Kobayashi GS, Pfaller MA. Medical microbiology. 3rd ed. St. Louis: Mosby; 1997.

8. Carmona H. Hepatite viral: novos vírus, novos métodos de diagnóstico e novas terapêuticas. Lisboa: Hospital de Santa Maria; 1999.

9. Direcção-Geral da Saúde. Vacina contra a hepatite B: actualização da vacinação gratuita de grupos de risco. Circular Normativa No. 15/DT. 2001.

10. Pereira M. Vacina anti-hepatite B: razões para a não vacinação dos profissionais de saúde. Rev Port Clin Geral 2000; 16: 15-20.

11. Ali NS, Jamal K, Qureshi R. Hepatitis B vaccination status and identification of risk factors for hepatitis B in health care workers. J Coll Physicians Surg Pak 2005 May; 15 (5): 257-60.

Os autores declararam não possuir conflitos de interesses

\section{ENDEREÇO PARA CORRESPONDÊNCIA}

Carla Susana Alves Gomes

Rua Adelino Amaro da Costa, $n^{\circ} 21,2^{\circ}$ Dto.

2720-002 Amadora

E-mail: gomessusana@hotmail.com

Recebido em 01/01/2008

Aceite para publicação em 09/11/2009 


\section{ABSTRACT}

\section{HEPATITIS B: VACCINATION COVERAGE OF HEALTH CARE PROFESSIONALS AT THE QUELUZ HEALTH CENTER}

Introduction: Hepatitis B is an important worldwide cause of morbidity and mortality, which transmission is related to occupational exposure. Vaccination against hepatitis B induces a high protection in vaccinated individuals, and it is therefore found included in the National Vaccination Plan and contemplated for risk groups, namely for health care professionals.

Objective: To establish the prevalence of the hepatitis B vaccination status in health care professionals at the Queluz Health Center.

Design: Descriptive, cross sectional study, observational

Setting: Queluz Health Center

Population: Health care professionals at risk.

Method: The data were obtained by computerized registered information from SINUS or by directly confirming each individual health bulletin and in the absence of these, by a questionnaire to the professionals. The following variables were considered: sex, age, profession, hepatitis B vaccination status.

Results: The population included 132 individuals, having obtained results for 104 of those individuals. Of these, $81,7 \%$ had completed vaccination, $5,8 \%$ incomplete and $12,5 \%$ had no vaccination whatsoever.

In relation to the professional groups, $92,1 \%$ of nurses had complete vaccination, $79,7 \%$ of physicians and $42,9 \%$ of nursing assistants.

The nursing assistants were the group with the highest absence of vaccination - $28,6 \%$, followed by the physicians - $16,9 \%$ and the nurses $-2,6 \%$.

Conclusion: It would be important to evaluate if these data really indicate low vaccination rates against Hepatitis B in health care professionals, by developing new studies including other health care professionals belonging to different Health Centers.

Given the clear indication for the vaccination of these professionals, the possibility of working with non-vaccinated individuals can be a way of spreading information in a perceivable way, emphasizing the benefits obtained with primary prevention. A future study to evaluate the motives that lead to non-vaccination can be considered.

Keyword: Vaccination; Hepatitis B; Health Care Professionals.

\section{ANEXO 1 \\ FOLHA DE REGISTO DE DADOS}

1. Profissão
a. Médico/a
b. Enfermeiro/a
c. Auxiliar de Acção Médica

2. Sexo
a. Feminino
b. Masculino

3. Idade

4. Estado Vacinal
a. Completo (3 doses)
b. Incompleto
c. Nunca se vacinou 